\title{
The Applications of Inductive Method in the Construction of Fault Trees MENG Qinghe ${ }^{1, a}$, SUN Qin ${ }^{2, b}$

\author{
${ }^{1}$ School of Aeronautics, Northwestern Polytechnical University, Xi'an 710072, China \\ ${ }^{2}$ School of Aeronautics, Northwestern Polytechnical University, Xi'an 710072, China \\ aemail: mengqh77@hotmail.com , bemail: sunqin@ nwpu.edu.cn
}

Keywords: function flow, inductive method, fault tree, system

\begin{abstract}
Fault tree analysis is a method for both reliability assessment and fault diagnosis. Generally, the deductive method is adopted in the construction of fault tree model. In this way, some of the undesired events may be omitted. This paper provides an inductive method. It is based on the working principle and system theory. Other than the deduction method, the method starts from the study of the failure modes of parts and then works up to analyze their influences on the subsystems. The relationships between the events and subsystems are illustrated by a nested structure. The induction sequence and process are also demonstrated. This method proved to be an effective way for the construction of fault tree.
\end{abstract}

\section{Introduction}

Fault tree analysis was first adopted in 1962 for the U.S. Air Force by Bell Telephone Laboratories for use with the Minuteman system. Then it was later extensively applied by the Boeing Company. [1, 2, 3, 5, 6] It's considered an effective method for both quantitative assessment and qualitative analysis. As a quantitative assessment method, the fault tree provides a mathematical model that is suitable for determining system safety and reliability. As a direct acyclic graph, it provides a logical and diagrammatic description of the causes of system fault in terms of component failures. It is a logic diagram based on statements which are either true or false, on or off, open or closed, good or bad, etc. In fact, as a logical variable, every node has only two values that are either true or false, but never partially true or false. Hence the Boolean algebra is a fitting companion for the fault tree. [1]

Generally, the construction of a fault tree starts from the top event that is identified the most undesired and then works down through the various branches. [7] Eventually the fault tree should end at the bottom events. The bottom events should be statistical independent each other. This method is named the deductive method. However, this method focus on the most undesirable events and some of the consequences or causes that are not the most undesirable but occur frequently may be omitted.

This paper presents an inductive method to construct the fault trees. The method manages to induce the consequences from the bottom events on basis of the clear understanding of the whole system. It focuses on the influence of the part failure on the subsystem. Analyzing the parts from in the upstream to the system in the downstream, repeating and continuing, the causal relationships between events are constructed gradually.

\section{The inductive method and function flow theory}

In order to construct a fault tree model that agrees well with the actual situation, the working principle must be understood very clearly and extensively. For this purpose, the functional diagram is essential to be read in detail on basis of working principle both in depth and in breadth.

By means of the functional diagram, the working process of the system that composed of subsystems and the parts, the inner boundary and the outer boundary of the system and the working flow charts of the parts are shown. It provides an overview of the system. The relationships and interactions between parts or systems are also shown. 
For example, Fig. 1 is a functional diagram of a lighting system and it illustrates the working principle of the system. From the diagram, it can be seen that the system is composed of seven parts that are the bulb $A$,battery $E_{1}, E_{2}$,inductor $J_{1}, J_{2}$, circuit breaker $K_{2}$, relay $K_{1}$ and switch $K_{3}$. There are three sets of circuits: circuit I ,circuit II and circuit III.Either circuit I or circuit II can provide the

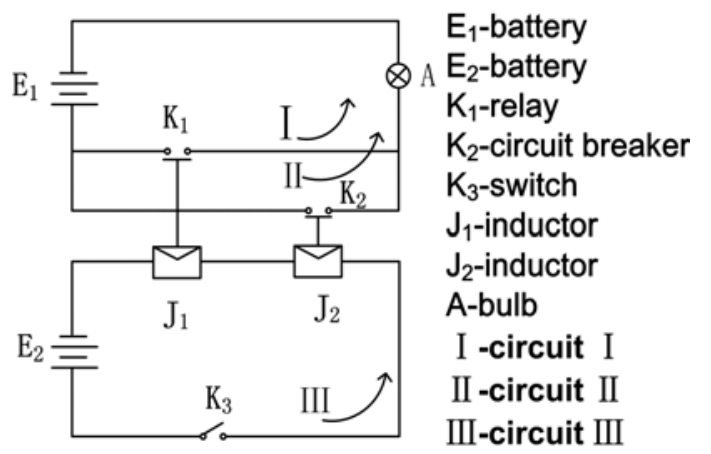

Fig.1 the functional diagram of a lighting system power supply to bulb A. They have a common battery $\mathrm{E}_{1}$. [5]

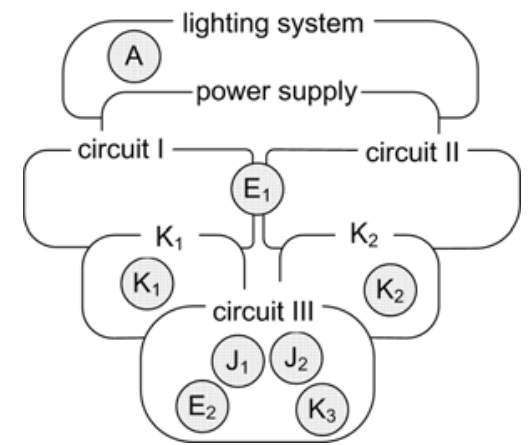

Fig.2 the system, subsystem and parts

As is shown in Fig.2, circuit $\mathrm{I}$ is controlled by $\mathrm{K}_{1}$ and $\mathrm{K}_{1}$ will be turned on if there is current in circuit III.Circuit II is controlled by $\mathrm{K}_{2}$ and $\mathrm{K}_{2}$ will be turned on if there is no current in circuit III. Circuit III is controlled by $\mathrm{K}_{3}$.

Generally, the function is actually fulfilled by a system that is composed of a chain of parts and not only the one part. Thus if some fault phenomenon occurs at some part, the real cause may not only lie in the last downstream part, but also attribute to its upstream parts that provide command, energy or feedback signal. [2] For example, if some fault occurs in circuit III,the real cause may lie in any part of circuit III that is relevant to some function. In other words, $E_{2}, J_{1}, J_{2}$ and $K_{3}$ should all be taken into account.

Thus in constructing the fault tree, it's important to divide the system into several subsystems that are independent of each other and find the failure modes of every component. And then analyze the influences of the part failure on the subsystem. On the other hand, the fault of subsystem is a component of another subsystem. Repeating the process in the others and continuing, all the intermediate events and top event can be found out.
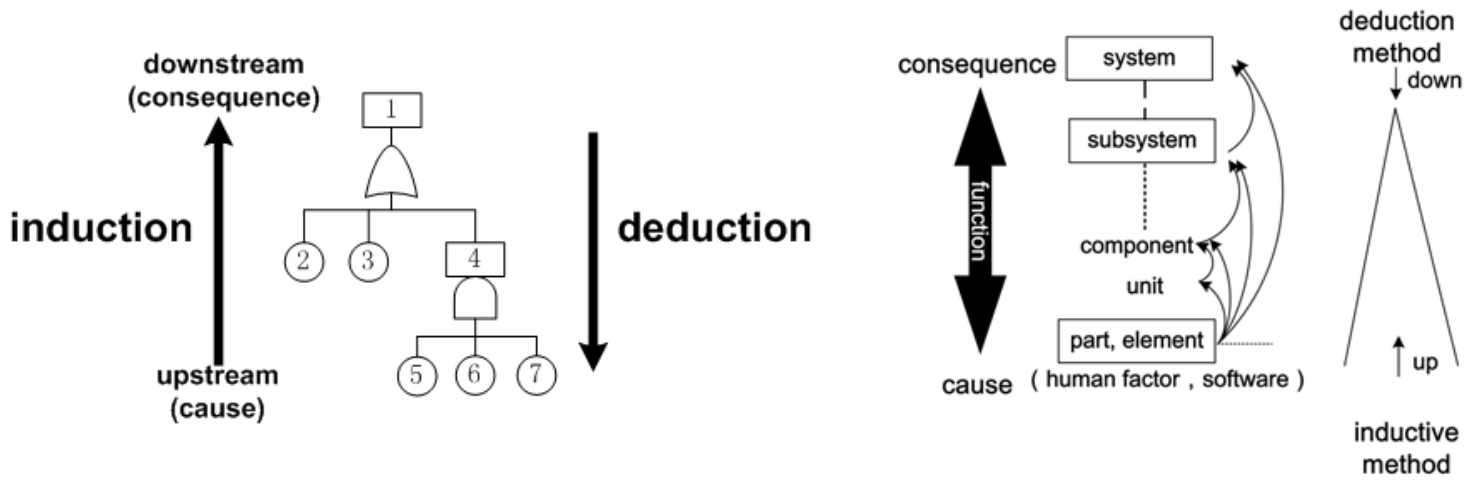

Fig. 3 the differences between the deductive method and inductive method

For this purpose, the function flow theory should be taken as guidance. The function flow means the energy, signal, information, quality and so on. If the function flow is input from the left side of the function system, there is some desirable function flow output from the right side, then the system works in normal. This is the main idea of the function flow theory. [2]

In fact, the system is not a real body but an event and so are the subsystems and components. The relationships are not the real structure in space but in logic and function. [4] Therefore in the fault tree, all the relevant elements should be taken into account such as the environmental factors, human factors, the failure of software and so on.[2]These elements are essential to the system. 


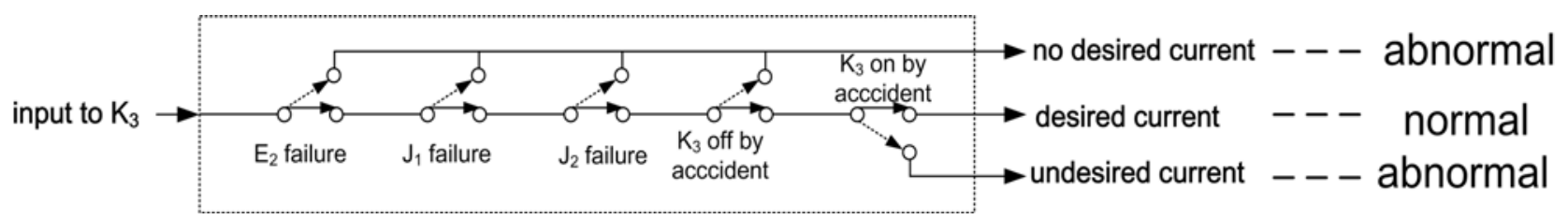

Fig.4 the function flow model of circuit III

The relationships of the components are determined by their functions and logics. For all the events has only two states, they can be compared to switches. So long as the state of every component is 0 , that means any of the failure modes doesn't happen, the function flow can pass through the model successfully. For example, if the switch $K_{3}$ is turned on ,there will be current in circuit III and if the switch $\mathrm{K}_{3}$ is turned off there will be no current in circuit III. Therefore, for circuit III, the function flow model should be shown as in Fig.4. In fact, every subsystem is a union of sufficient and necessary conditions for the function.

If the state of some component is 1 , that means true, the function flow may be cut off and various undesirable events may occur. In fact, most of the parts work independently, therefore their failure modes are independent of each other, but have an influence on the subsystem. Besides, according to No Magic Rule, if some part fails, the consequence will be transmitted to the downstream parts, assuming that the other parts are normal. Under the wrong consequences, the downstream parts may be forced to work abnormally, though the parts themselves are well. [5] Hence the consequences of the system III should be shown as Fig. 5.

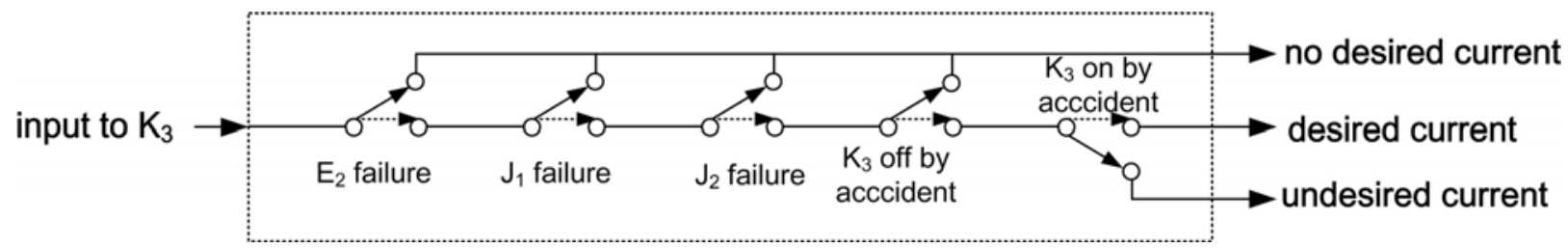

Fig.5 the different consequences the events in circuit III bring about

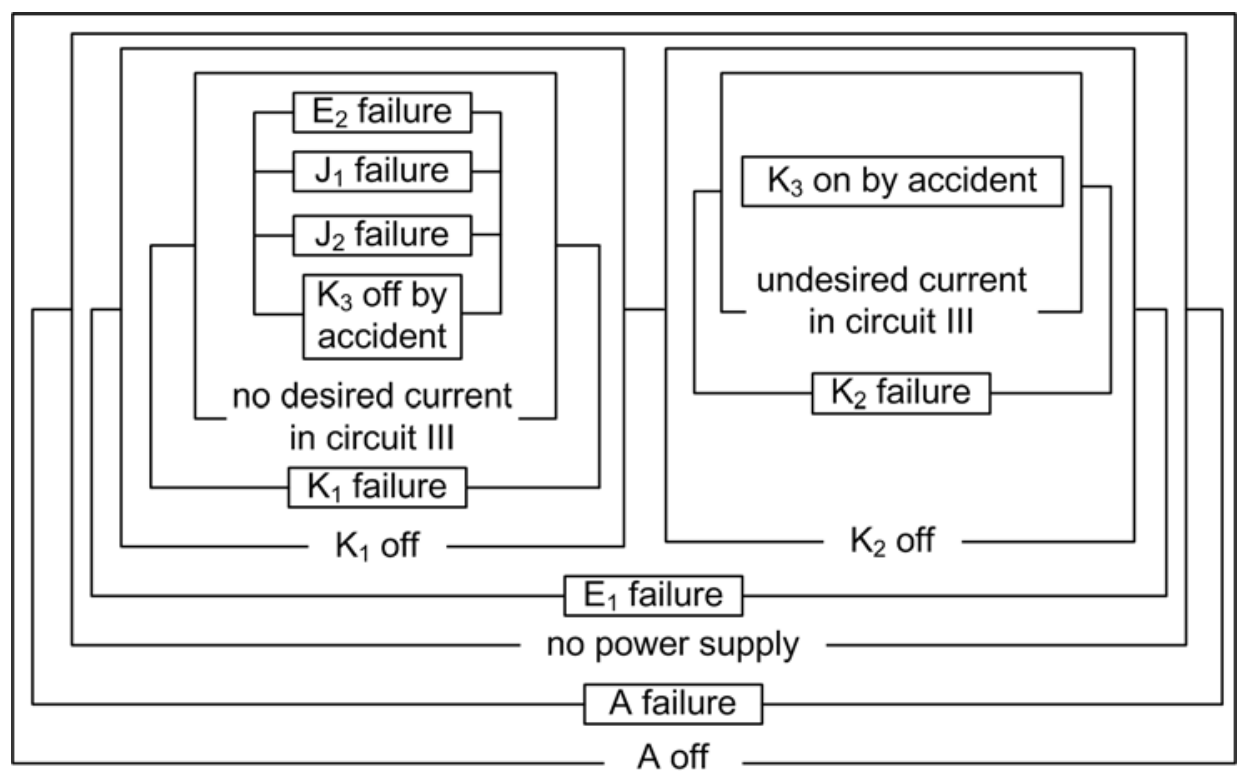

Fig.6 the nested structure of the fault tree system

\section{Application to an example}

According to the function diagram in Fig. 1, a fault tree can be constructed step by step. The working principle and control flow chart has been discussed above. It can be seen that the fault tree 
is in fact a nested structure as is shown in Fig. 6. The units in the fault tree are undesirable events. The subsystems and the system is a nested structure. Thus the analysis should start from the inner part to the exterior subsystem and then analyze from one subsystem to another and end at the top event. The consequence of a subsystem is a cause of another subsystem and one fault of a subsystem may lead to a chain reaction in another subsystem. Hence the analysis should start from one subsystem to another and the consequence of the subsystem is in fact an intermediate event in the fault tree.

The process of induction is shown in Fig. 7. It's in fact a demonstration of causal induction flow chart. The induction process and steps can be seen clearly and it's easy to be understood and needless to be explained.

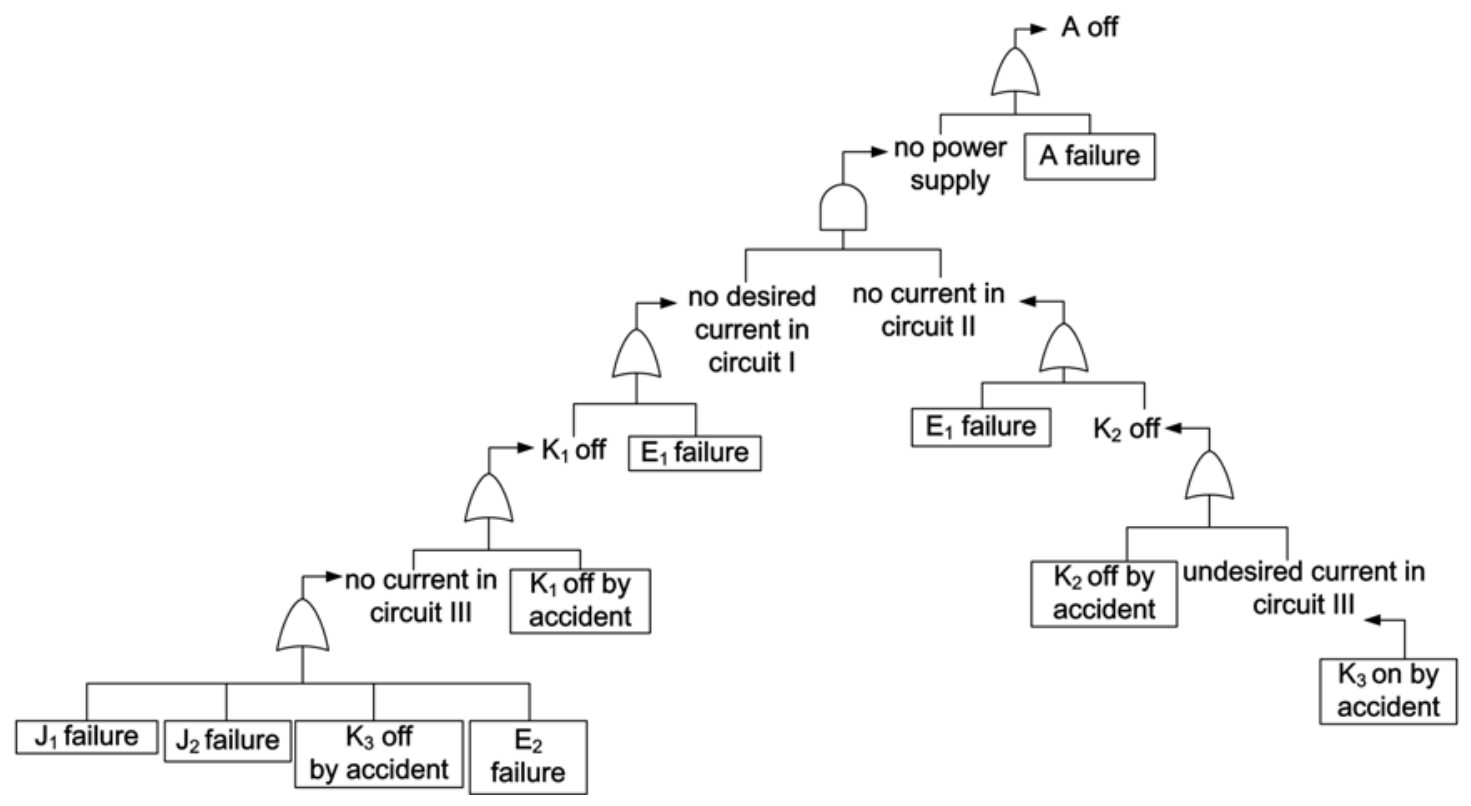

Fig.7 the flow chart of causal induction

Tracing back in inverse order, the fault tree can be achieved, as is shown in Fig. 8.

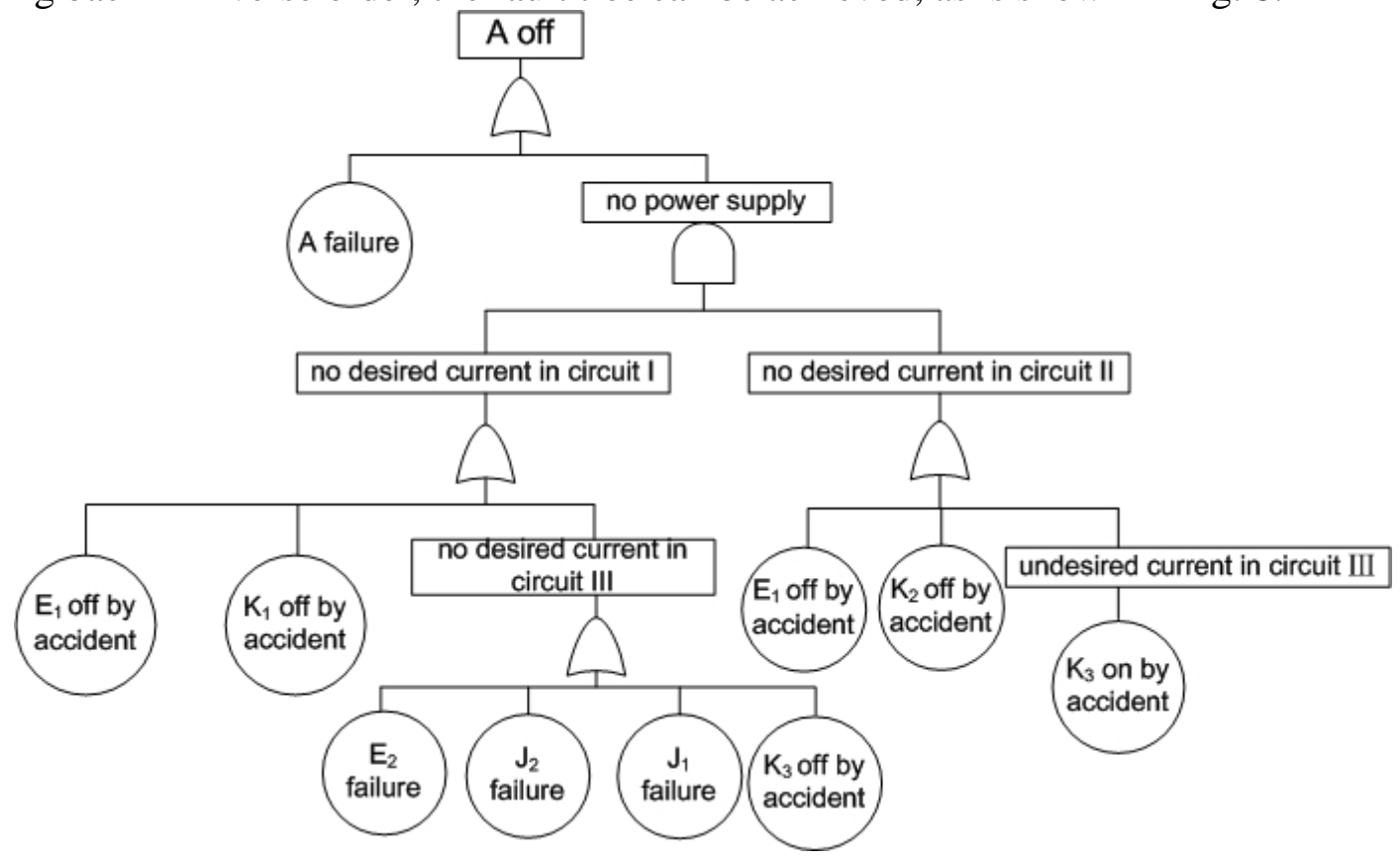

Fig.8 the fault tree achieved 


\section{Summary}

Compared with the example in the books, the fault tree is almost identical. This proves that this method is right and effective. In spite of the difference between the forms of the fault trees, their Boolean logic expressions are identical after simplified. The fault tree is just the graphic presentation of the Boolean algebra expression, so the form is not important. In fact, after simplified, the Boolean expression is just a top event expression in terms of the bottom events and no intermediate events. This method focuses on the influence of component failure modes on the subsystem. It's in fact an induction to the direct consequence so it can also be named the direct consequence method. No matter how complex the relationships are among the events, the most important is to divide the system into subsystems by their functions correctly and make a study on their causal relationships. The subsystems should be independent of each other. Finally, the function flow theory and system idea must be understood clearly and applied to the fault tree construction.

\section{References}

[1] WALDEMAR F. LARSEN: Fault Tree Analysis, Technical report 4556, New Jersey, January 1974, p. 2

[2] Shiomi hiroshi, Shimaoka atsushi, Ishiyama takayuki, XU Fengzhang, GAO Jinzhong: the application of FMEA and FTA, China Machine Press ( 1987 )

[3] ZHU Jizhou: the Principle and Application of FTA, Xi'an Jiaotong University Press (1989)

[4] W. E. Vizili, SHU Songgui, TANG Xinqing: Handbook of FTA, Atomic Energy Press (1987), chapter 5

[5] MAO Tiancheng: the application of FTA in Diagnosis of the Automatic Control System of Ships, Dalian Maritime University Press (2000)

[6] P. L. Clemens: Fault Tree Analysis, JACOBS SVERDRUP (2002)

[7] Joanne Bechta Dugan: Fault Tree Analysis and Computer-Based Systems, RELIABILITY and MAINTAINABILITY Symposium 